CORRECTION

\title{
Correction to: Divorce among European and Mexican immigrants in the U.S.
}

\section{Christina A. Houseworth $\mathbb{D}^{1} \cdot$ Barry R. Chiswick ${ }^{2}$}

Published online: 14 May 2019

(C) Springer Science+Business Media, LLC, part of Springer Nature 2019

\section{Correction to: Rev Econ Household}

https://doi.org/10.1007/s11150-019-09447-0

The original version of this article unfortunately contained few errors in tables. In Tables 2a, 2b, 3a and 3b, the "Percent of sample" and "Percent spouse absent" should be in decimal in order to be consistent. In Tables 4 and 5, the first row should read as "Age of marriage" instead of "Percent of sample" and the "Sample size" was incorrect.

The corrected tables are presented below:

The original article can be found online at https://doi.org/10.1007/s11150-019-09447-0

Christina A. Houseworth

houseworth@hws.edu

$\triangle$ Barry R. Chiswick

brchis@gwu.edu

1 Department of Economics, Hobart \& William Smith Colleges, 312 Stern Hall, Pulteney Street, Geneva, NY 14456, USA

2 Department of Economics, George Washington University, Monroe Hall, Room 340, $2115 \mathrm{G}$ Street, NW, Washington, DC 20052, USA 
Table 2a Summary statistics European men

\begin{tabular}{|c|c|c|c|c|}
\hline & \multicolumn{2}{|l|}{ Married } & \multicolumn{2}{|l|}{ Divorced } \\
\hline & Foreign born & Native born & Foreign born & Native born \\
\hline Percent of sample & 0.92 & 0.87 & 0.08 & 0.13 \\
\hline Percent spouse absent & 0.04 & 0.02 & & \\
\hline Age at marriage & 27.37 & 26.41 & 27.10 & 25.76 \\
\hline Married prior to migration & 0.46 & & 0.36 & \\
\hline Country of origin divorce rate & 1.99 & & 1.99 & \\
\hline Linguistic closeness & 0.61 & & 0.67 & \\
\hline State divorce rate & 8.30 & 9.01 & 8.49 & 9.16 \\
\hline Self reported english only or very well & 0.71 & 0.98 & 0.81 & 0.99 \\
\hline Self reported no english or poor english & 0.09 & 0.00 & 0.05 & 0.00 \\
\hline Migrated between $0-13$ & 0.17 & & 0.25 & \\
\hline Migrated between 14-18 & 0.09 & & 0.09 & \\
\hline Migrated between 19-25 & 0.24 & & 0.26 & \\
\hline Migrated between $26-35$ & 0.35 & & 0.29 & \\
\hline Migrated between $36-45$ & 0.13 & & 0.08 & \\
\hline Migrated at 46 or after & 0.02 & & 0.02 & \\
\hline Groupsize & 1.05 & 5.17 & 1.33 & 5.59 \\
\hline Availability ratio & 1.11 & 0.97 & 0.90 & 0.90 \\
\hline Age & 42.23 & 41.85 & 44.06 & 44.35 \\
\hline Years of education & 14.84 & 14.65 & 14.45 & 13.53 \\
\hline Children under 6 & 0.17 & 0.16 & 0.04 & 0.04 \\
\hline Children between 6 and 17 & 0.33 & 0.35 & 0.13 & 0.19 \\
\hline Children in both age groups & 0.12 & 0.13 & 0.02 & 0.03 \\
\hline Sample size & 20,491 & 535,319 & 1847 & 82,887 \\
\hline
\end{tabular}

Source: 2010-2014 American Community Survey

Groupsize is in thousands. Variables are defined in Table 7 
Table 2b Summary statistics European women

\begin{tabular}{|c|c|c|c|c|}
\hline & \multicolumn{2}{|l|}{ Married } & \multicolumn{2}{|l|}{ Divorced } \\
\hline & Foreign born & Native born & Foreign born & Native born \\
\hline Percent of sample & 0.88 & 0.85 & 0.12 & 0.15 \\
\hline Percent spouse absent & 0.04 & 0.02 & & \\
\hline Age at marriage & 24.94 & 24.72 & 24.41 & 23.66 \\
\hline Married prior to migration & 0.54 & & 0.49 & \\
\hline Country of origin divorce rate & 2.09 & & 2.19 & \\
\hline Linguistic closeness & 0.58 & & 0.62 & \\
\hline State divorce rate & 8.93 & 9.40 & 9.09 & 9.54 \\
\hline Self reported english only or very well & 0.71 & 0.98 & 0.81 & 0.99 \\
\hline Self reported no english or poor english & 0.08 & 0.00 & 0.05 & 0.00 \\
\hline Migrated between $0-13$ & 0.18 & & 0.21 & \\
\hline Migrated between $14-18$ & 0.09 & & 0.09 & \\
\hline Migrated between 19-25 & 0.30 & & 0.34 & \\
\hline Migrated between $26-35$ & 0.32 & & 0.27 & \\
\hline Migrated between $36-45$ & 0.10 & & 0.08 & \\
\hline Migrated at 46 or after & 0.01 & & 0.01 & \\
\hline Groupsize & 1.26 & 5.22 & 1.54 & 5.12 \\
\hline Availability ratio & 1.26 & 1.17 & 0.99 & 1.09 \\
\hline Age & 41.01 & 41.12 & 43.77 & 43.75 \\
\hline Years of education & 14.69 & 14.83 & 14.68 & 14.08 \\
\hline Children under 6 & 0.16 & 0.16 & 0.06 & 0.06 \\
\hline Children between 6 and 17 & 0.32 & 0.34 & 0.28 & 0.32 \\
\hline Children in both age groups & 0.12 & 0.12 & 0.03 & 0.06 \\
\hline Sample size & 23,309 & 574,494 & 3093 & 102,320 \\
\hline
\end{tabular}

Source: 2010-2014 American Community Survey

Groupsize is in thousands. Variables are defined in Table 7 
Table 3a Summary statistics mexican men

\begin{tabular}{|c|c|c|c|c|}
\hline & \multicolumn{2}{|l|}{ Married } & \multicolumn{2}{|l|}{ Divorced } \\
\hline & Foreign born & Native born & Foreign born & Native born \\
\hline Percent of sample & 0.94 & 0.91 & 0.06 & 0.09 \\
\hline Percent spouse absent & 0.11 & 0.09 & & \\
\hline Age at marriage & 25.19 & 25.18 & 25.08 & 24.69 \\
\hline Married prior to migration & 0.29 & & 0.26 & \\
\hline State divorce rate & 9.13 & 9.18 & 9.22 & 9.30 \\
\hline Self reported english only or very well & 0.26 & 0.46 & 0.38 & 0.68 \\
\hline Self reported no english or poor english & 0.44 & 0.31 & 0.32 & 0.15 \\
\hline Migrated between $0-13$ & 0.18 & & 0.26 & \\
\hline Migrated between 14-18 & 0.29 & & 0.25 & \\
\hline Migrated between 19-25 & 0.32 & & 0.28 & \\
\hline Migrated between 26-35 & 0.17 & & 0.16 & \\
\hline Migrated between $36-45$ & 0.04 & & 0.05 & \\
\hline Migrated at 46 or after & 0.01 & & 0.01 & \\
\hline Groupsize & 2.02 & 2.07 & 2.02 & 2.14 \\
\hline Availability ratio & 0.85 & 0.86 & 0.84 & 0.85 \\
\hline Age & 40.02 & 39.13 & 42.08 & 41.64 \\
\hline Years of education & 9.37 & 10.47 & 10.16 & 11.42 \\
\hline Children under 6 & 0.12 & 0.15 & 0.08 & 0.07 \\
\hline Children between 6 and 17 & 0.39 & 0.36 & 0.25 & 0.24 \\
\hline Children in both age groups & 0.30 & 0.28 & 0.13 & 0.11 \\
\hline Sample size & 73,509 & 102,223 & 4968 & 10,473 \\
\hline
\end{tabular}

Source: 2010-2014 American Community Survey

Groupsize is in thousands. Variables are defined in Table 7 
Table 3b Summary statistics Mexican women

\begin{tabular}{|c|c|c|c|c|}
\hline & \multicolumn{2}{|l|}{ Married } & \multicolumn{2}{|l|}{ Divorced } \\
\hline & Foreign born & Native born & Foreign born & Native born \\
\hline Percent of sample & 0.92 & 0.88 & 0.08 & 0.12 \\
\hline Percent spouse absent & 0.07 & 0.08 & & \\
\hline Age at marriage & 23.31 & 23.36 & 22.89 & 22.63 \\
\hline Married prior to migration & 0.45 & & 0.37 & \\
\hline State divorce rate & 10.04 & 10.11 & 10.05 & 10.26 \\
\hline Self reported english only or very well & 0.24 & 0.48 & 0.37 & 0.69 \\
\hline Self reported no english or poor english & 0.56 & 0.37 & 0.39 & 0.18 \\
\hline Migrated between $0-13$ & 0.19 & & 0.29 & \\
\hline Migrated between $14-18$ & 0.21 & & 0.22 & \\
\hline Migrated between $19-25$ & 0.34 & & 0.26 & \\
\hline Migrated between $26-35$ & 0.20 & & 0.17 & \\
\hline Migrated between $36-45$ & 0.05 & & 0.06 & \\
\hline Migrated at 46 or after & 0.01 & & 0.01 & \\
\hline Groupsize & 2.00 & 2.06 & 1.96 & 2.11 \\
\hline Availability ratio & 1.24 & 1.23 & 1.18 & 1.19 \\
\hline Age & 39.18 & 38.05 & 42.08 & 40.99 \\
\hline Years of education & 9.68 & 10.94 & 10.57 & 11.90 \\
\hline Children under 6 & 0.12 & 0.15 & 0.08 & 0.08 \\
\hline Children between 6 and 17 & 0.40 & 0.37 & 0.42 & 0.39 \\
\hline Children in both age groups & 0.30 & 0.28 & 0.22 & 0.19 \\
\hline Sample size & 71,832 & 107,456 & 6478 & 14,245 \\
\hline
\end{tabular}

Source: 2010-2014 American Community Survey

Groupsize is in thousands. Variables are defined in Table 7 
Table 4 Selected variables from linear regression results for immigrants and the native born of European ancestry, married only once (Married $=0$ and Divorced $=1$ )

\begin{tabular}{|c|c|c|c|c|}
\hline & \multicolumn{2}{|l|}{ Men } & \multicolumn{2}{|l|}{ Women } \\
\hline & Foreign born & Native born & Foreign born & Native born \\
\hline Age of marriage & -0.002 & $-0.011 * * *$ & -0.004 & $-0.014 * * *$ \\
\hline Married prior to migration & $-0.034 * * *$ & - & $-0.026 * *$ & - \\
\hline Country of origin divorce rate & $0.007 * *$ & - & $0.016 * * *$ & - \\
\hline COO high divorce $\mathrm{X}$ groupsize & 0.003 & - & $0.007 * * *$ & - \\
\hline Linguistic closeness & $0.062 * *$ & - & $0.044^{*}$ & - \\
\hline State divorce rate & 0.001 & $0.004 * * *$ & 0.001 & $0.004 * * *$ \\
\hline $\begin{array}{l}\text { Self reported english only or } \\
\text { very well }\end{array}$ & $0.033 * * *$ & $0.087 * * *$ & $0.042 * * *$ & $0.090 * * *$ \\
\hline $\begin{array}{l}\text { Self reported no english or poor } \\
\text { english }\end{array}$ & $-0.018^{*}$ & $-0.041 * * *$ & -0.009 & $-0.029 * * *$ \\
\hline Migrated between $0-13$ & -0.004 & - & -0.015 & - \\
\hline Migrated between $14-18$ & -0.003 & - & $-0.024 *$ & - \\
\hline Migrated between $26-35$ & -0.012 & - & $-0.036 * * *$ & - \\
\hline Migrated between $36-45$ & $-0.031^{*}$ & - & $-0.058 * * *$ & - \\
\hline Migrated at 46 or after & -0.016 & - & -0.033 & - \\
\hline Groupsize & -0.033 & $-0.004 * * *$ & $-0.064 * *$ & $-0.004 * * *$ \\
\hline Availability ratio & $-0.022 * * *$ & $-0.033 * * *$ & $-0.031 * * *$ & $-0.034 * * *$ \\
\hline Age & $0.013 * * *$ & $0.022 * * *$ & $0.033 * * *$ & $0.039 * * *$ \\
\hline Years of education & 0.000 & $-0.019 * * *$ & $0.015^{* * *}$ & $-0.011 * * *$ \\
\hline Children under 6 & - & - & $-0.111^{* * *}$ & $-0.121 * * *$ \\
\hline Children between 6 and 17 & - & - & $-0.089 * * *$ & $-0.091 * * *$ \\
\hline Children in both age groups & - & - & $-0.151 * * *$ & $-0.143 * * *$ \\
\hline Sample size & 22,324 & 627,338 & 26,379 & 684,662 \\
\hline $\mathrm{R} 2$ & 0.030 & 0.068 & 0.046 & 0.057 \\
\hline
\end{tabular}

Source: 2010-2014 American Community Survey

Sample restricted to persons age 18-55, excludes those widowed, separated, and never married. Migration related variables not entered for native born. Controls for the year of the ACS data, region in the U.S., age at marriage squared, age squared, and education squared are included. Group size is in ten thousands. Person weights provided by the ACS were utilized. Variables are defined in Table 7. Calculated using robust standard errors, clustered by state and PUMA

$* p<0.05$. *** $p<0.01$. ***p $<0.001$ 
Table 5 Selected variables from linear regression results for immigrants and the native born of Mexican ancestry, married only once (Married $=0$ and Divorced $=1$ )

\begin{tabular}{|c|c|c|c|c|}
\hline & \multicolumn{2}{|l|}{ Men } & \multicolumn{2}{|l|}{ Women } \\
\hline & Foreign born & Native born & Foreign born & Native born \\
\hline Age of marriage & $-0.005^{* * *}$ & $-0.014 * * *$ & $-0.006^{* * *}$ & $-0.014 * * *$ \\
\hline Married prior to migration & $-0.019 * * *$ & - & $-0.026 * * *$ & - \\
\hline State divorce rate & $0.003 * *$ & $0.007 * * *$ & $0.003^{*}$ & $0.007 * * *$ \\
\hline $\begin{array}{l}\text { Self reported english only or } \\
\text { very well }\end{array}$ & $0.020^{* * *}$ & $0.075^{* * *}$ & $0.024 * * *$ & $0.075 * * *$ \\
\hline $\begin{array}{l}\text { Self reported no english or poor } \\
\text { english }\end{array}$ & -0.005 & $-0.035 * * *$ & $-0.019 * * *$ & $-0.035 * * *$ \\
\hline Migrated between 0-13 & $0.027 * * *$ & - & $0.027 * * *$ & - \\
\hline Migrated between $14-18$ & 0.001 & - & $0.018 * * *$ & - \\
\hline Migrated between 26-35 & $0.010^{*}$ & - & 0.006 & - \\
\hline Migrated between $36-45$ & 0.011 & - & 0.013 & - \\
\hline Migrated at 46 or after & 0.023 & - & 0.008 & - \\
\hline Groupsize & -0.012 & -0.001 & -0.002 & 0.003 \\
\hline Availability ratio & $-0.023 * * *$ & 0.006 & $-0.017 * * *$ & $-0.019 * * *$ \\
\hline Age & $0.010 * * *$ & $0.016 * * *$ & $0.016 * * *$ & $0.025 * * *$ \\
\hline Years of education & -0.001 & $0.009 * * *$ & 0.002 & $0.011^{* * *}$ \\
\hline Children under 6 & - & - & $-0.037 * * *$ & $-0.087 * * *$ \\
\hline Children between 6 and 17 & - & - & $-0.038 * * *$ & $-0.074 * * *$ \\
\hline Children in both age groups & - & - & $-0.041 * * *$ & $-0.087 * * *$ \\
\hline Sample size & 74,803 & 130,759 & 74,739 & 137,444 \\
\hline R2 & 0.015 & 0.037 & 0.042 & 0.062 \\
\hline
\end{tabular}

Source: 2010-2014 American Community Survey

Sample restricted to persons age 18-55, excludes those widowed, separated, and never married. Migration related variables not entered for native born. Controls for the year of the ACS data, region in the U.S., age at marriage squared, age squared, and education squared are included. Group size is in ten thousands. Person weights provided by the ACS were utilized. Variables are defined in Table 7. Calculated using robust standard errors, clustered by state and PUMA

$* p<0.05 . * * p<0.01 . * * * p<0.001$

The original article has been corrected. 